\title{
Le Mécanisme pour un Développement Propre : esquisse géo-environnementale de la participation du Canada
}

\author{
MoÏse TSAYEM DEMAZE \\ Maître de conférences en géographie à l'Université du Maine \\ UMR CNRS 6590 Espaces et sociétés (ESO Le Mans) \\ Moise.Tsayem_Demaze@univ-lemans.fr
}

\section{Résumé}

Institué par le Protocole de Kyoto pour réduire les émissions de gaz à effet de serre (EGES), le Mécanisme pour un Développement Propre (MDP) a engendré une nouvelle coopération NordSud illustrée dans cet article avec le cas du Canada associé aux pays en développement. Les données utilisées montrent qu'en janvier 2011, la Chine, l'Inde et le Brésil sont les principaux pays hôtes des projets MDP, alors que le Royaume Uni, la Suisse, le Japon et les Pays-Bas, sont les principaux pays investisseurs. La participation du Canada est quasi médiane et apparaît faible ( $1,6 \%$ des projets acceptés pour $9,9 \%$ des crédits carbone délivrés). Elle lui permet une présence dans des projets MDP à l'étranger (en Chine et au Brésil par exemple), et lui offre l'opportunité de prendre part aux marchés carbone et au transfert de technologies dans divers secteurs environnementaux, comme les industries énergétiques, le traitement et l'élimination des déchets, l'agriculture. Cependant, le MDP n'apparaît pas comme un élément essentiel de la politique canadienne de réduction des EGES, car l'État canadien, qui accordait peu d'importance au Protocole de Kyoto, se situe déjà dans une perspective post ou hors Kyoto. L'article rend compte de la géographie engendrée par le MDP et propose une esquisse cartographique de la participation du Canada à ce mécanisme.

\section{Abstract. The Clean Development Mechanism : geo-environmental appraisal of Canada's participation}

Established by the Kyoto Protocol to reduce greenhouse gases emissions, the CDM mechanism has led to a new North-South cooperation that will be developed in this paper with the case of Canada associated to developing countries. The data we used show that, in January 2011, China, India and Brazil were leaders of developing countries participating in the CDM, whereas for developed countries, United Kingdom, Switzerland, Japan and Netherlands were leaders. Canada's participation is nearly median and appears low (1.6\% of accepted projects for $9.9 \%$ of carbon credits issued). It offers to him an abroad presence in CDM projects (in China and Brazil for example) and the opportunity to take part in carbon markets and in technology transfer in various environmental sectors, such as energy industries, waste treatment disposal, agriculture. However, the CDM is not an essential element of Canada's policy in reducing greenhouse gases emissions, since the Canadian government seems to attach little importance to the Kyoto Protocol, lying in a post or out Kyoto perspective. The article describes the geography created by $\mathrm{CDM}$ and provides an outline map of Canada's participation in this mechanism.

Mots-clés : mécanisme pour un développement propre, Protocole de Kyoto, changements climatiques, gaz à effet de serre, Canada.

Keywords: clean development mechanism, Kyoto Protocol, climatic change, greenhouse gases, Canada. 


\section{Introduction}

La lutte contre le réchauffement de la Terre est devenue une préoccupation primordiale de la communauté internationale (Banque Mondiale 2009; Mens 2008 ; Denis 2007 ; PNUD 2007 ; Barral 2006). Le Protocole de Kyoto, adopté en 1997 en complément de la Convention des Nations unies sur les changements climatiques ${ }^{1}$, constitue le cadre international de l'organisation et de la mise en œuvre de la participation des États à cette lutte (Tsayem, 2009a; 2009b). Elle porte essentiellement sur la réduction des émissions de gaz à effet de serre ${ }^{2}$ (EGES) et repose sur le principe des responsabilités communes mais différenciées (Lavallée, 2010). Selon ce principe, les pays développés, appelés pays de l'annexe 1 de la Convention sur les changements climatiques, sont considérés comme principaux responsables du réchauffement de la Terre (contrairement aux pays en développement, qui sont appelés pays non annexe 1). Sur la base de ce principe, les pays développés sont tenus de réduire leurs EGES, tandis que les pays en développement, y compris les pays émergents comme la Chine, l'Inde et le Brésil, en sont dispensés. La méthode choisie est l'attribution de quotas d'émissions permettant d'aboutir globalement à une réduction de $5 \%$ des EGES en 2012 par rapport aux EGES en 1990 (article 3 du Protocole de Kyoto). La période 2008-2012 est retenue comme première phase (Kyoto 1) de l'application ou du respect des réductions des EGES ( $8 \%$ de réduction pour l'Union européenne, $7 \%$ pour les Etats-Unis ${ }^{3}, 6 \%$ pour le Japon ainsi que pour le Canada, etc.).

Pour permettre aux pays développés de réduire leurs EGES, le Protocole de Kyoto a prévu trois mécanismes dits de flexibilité (UNFCCC, 1998) : les échanges ou le commerce des droits d'EGES entre les pays développés (marchés des quotas d'émissions ou permis transférables), la mise en œuvre conjointe (accords ou ententes permettant aux pays de l'annexe 1 de réduire leurs EGES notamment dans des pays d'Europe du Centre et de l'Est), et le Mécanisme pour un Développement Propre (MDP). Le MDP offre aux pays développés l'opportunité de remplir une partie de leurs engagements de réduction des EGES en finançant dans des pays en développement des projets à technologie "propre », c'est-à-dire peu productrice ou peu émettrice de ces gaz (Hall et al., 2008 ; Barral, 2006). Ainsi, les pays en développement se développent «proprement» et les pays développés ont la possibilité d'obtenir des quantités d'EGES correspondant au volume évité ou économisé dans des pays en développement grâce à l'utilisation de la technologie «propre » venant des pays développés.

L'objectif principal de cet article est de fournir des éléments de compréhension du fonctionnement du MDP et de ses dimensions géographiques et environnementales en s'appuyant sur l'exemple d'un pays de l'annexe 1 pour montrer comment ce pays participe à ce mécanisme. Le choix a porté opportunément sur le Canada ${ }^{4}$. Il s'agit d'une première étude de cas qui illustre les traitements effectués sur les données du $\mathrm{MDP}^{5}$. L'esquisse géo-environnementale dont il est question dans cette étude se rapporte à l'aspect géographique : réseaux associant le Canada aux

\footnotetext{
${ }^{1}$ Convention adoptée en 1992 lors de la Conférence des Nations unies sur l'Environnement et le Développement (conférence organisée à Rio de Janeiro au Brésil et baptisée Sommet de la Terre).

${ }^{2}$ La lutte contre le réchauffement de la Terre s'est focalisée jusqu'à présent sur l'atténuation ou la mitigation, consistant essentiellement en la réduction des EGES. L'adaptation est de plus en plus prônée (GIEC, 2007).

${ }^{3}$ qui ont signé le protocole mais ne l'ont pas ratifié, ce qui les affranchit de tout engagement contraignant.

${ }^{4}$ suite à l'obtention d'une bourse de recherche en études canadiennes (BREC) attribuée par le gouvernement du Canada (Affaires étrangères et Commerce international Canada) pour l'année universitaire 2010-2011.

${ }^{5}$ D'autres études de cas sont en cours ; elles portent sur le Royaume Uni, les Pays-Bas, la France et la Belgique. Elles ont donné lieu à un article proposé à la revue Mappemonde.
} 
pays en développement qui sont hôtes des projets MDP auxquels participe le Canada. L'esquisse concerne aussi l'aspect environnemental : secteurs environnementaux pour lesquels le Canada contribue au transfert de technologies "propres » dans des pays en développement, quantité d'émissions réduites octroyées pour les projets auxquels participe le Canada. Cette esquisse, essentiellement cartographique, met le Canada en lien avec les pays dans lesquels il participe aux projets MDP. L'article apporte des éléments de réponse aux questions suivantes : quels sont les pays en développement dans lesquels le Canada est impliqué dans la réalisation des projets MDP? Quelle est la quantité de crédits carbone délivrés pour les projets auxquels le Canada participe? Quels sont les secteurs environnementaux concernés par les projets MDP dans lesquels le Canada participe? Quelles sont les quantités de crédits carbone engendrées par ces projets? Comment la participation du Canada aux projets MDP dans les pays en développement s'inscritelle dans la politique canadienne des relations internationales?

Les résultats présentés dans cet article sont issus du traitement des données obtenues à la suite des requêtes effectuées en janvier 2011 sur le site Internet du conseil exécutif du MDP. Ce conseil est un organe ad hoc des Nations unies au sein de la Convention sur les changements climatiques. Il répertorie sur son site Internet l'ensemble des données relatives aux projets MDP (UNFCC, 2011a) : type de projets, pays impliqués, quantités d'EGES à éviter, etc. Des données complémentaires ont été obtenues auprès du RISOE Centre on Energy, Climate and Sustainable Development (UNEP RISOE, 2011a). C'est une structure de recherche qui assiste le Programme des Nations unies pour l'Environnement (PNUE) en matière de prise en compte de l'environnement et du développement dans la planification des politiques énergétiques dans le monde, et en particulier dans les pays en développement. Le RISOE Centre prétraite les données qui émanent du conseil exécutif du MDP. Il trie et regroupe les projets MDP par pays, par région géographique, par type de projets et par quantité d'EGES, etc ${ }^{6}$.

Le traitement des données a été précédé par un séjour de recherche au Canada, en juillet $2010^{7}$. Au cours de ce séjour, une recherche bibliographique a été effectuée à l'université Laval, et des entretiens ont eu lieu avec des personnes en charge de la participation du Canada au MDP et au protocole de Kyoto (Bureau Canadien du MDP et de l'Application Conjointe, Environnement Canada) et avec un expert travaillant pour la Fondation David Suzuki. Cette fondation collabore avec le gouvernement canadien, notamment sur les études scientifiques permettant d'alimenter les politiques énergétiques et la lutte contre le changement climatique.

Après avoir présenté les fondements et le fonctionnement du MDP pour en fournir un état global, cet article esquisse la participation du Canada à ce mécanisme, en illustrant d'abord les aspects géographiques puis les aspects environnementaux. Ces deux aspects permettent d'alimenter la réflexion sur les dimensions internationales et environnementales de la mise en œuvre du MDP et de la participation du Canada à ce mécanisme.

\section{Fondements et fonctionnement du MDP}

Le Protocole de Kyoto a ciblé la réduction des EGES par les États comme un moyen de lutte contre les changements climatiques. Outre les réductions domestiques, c'est-à-dire à l'intérieur de chaque pays concerné (pays développés dits de l'annexe 1), le Protocole de Kyoto a prévu, par le

\footnotetext{
${ }^{6}$ Voir http://cdmpipeline.org/ (consulté le 6 juillet 2012)

${ }^{7}$ Grâce à l'obtention d'une bourse de recherche en études canadiennes (BREC)
} 
MDP, que des réductions soient possibles dans des pays non annexe 1 (pays en développement, y compris les pays émergents) grâce à la coopération entre les pays développés et les pays en développement. Ainsi, même s'ils ne sont tenus à aucun engagement chiffré de réduction de leurs EGES, les pays en développement sont perçus dans le Protocole de Kyoto comme des lieux où des réductions peuvent être effectuées en coopération avec les pays développés. Par le MDP, la réduction des EGES dans les pays en développement est envisagée comme une forme d'aide au « développement durable» de ces pays en développement. Bien que cette référence au développement durable ne soit pas explicitée dans le Protocole de Kyoto, on peut supposer que la réduction des EGES dans les pays en développement soit destinée à permette à ces pays de se développer sans émettre trop de GES, de manière à ce que, contrairement au développement des pays aujourd'hui développés, le développement des pays en développement se fasse sans dégrader davantage le climat de la Terre. Cependant, la possibilité offerte aux pays développés de réduire les EGES dans les pays en développement grâce au MDP apparaît aussi et peut-être surtout comme une aide à ces pays développés. D'après l'article 12 du Protocole de Kyoto, « l'objet du mécanisme pour un développement «propre» est d'aider les Parties [pays] ne figurant pas à l'annexe 1 à parvenir à un développement durable ainsi qu'à contribuer à l'objectif ultime de la Convention, et d'aider les Parties [pays] visées à l'annexe 1 à remplir leurs engagements chiffrés de limitation et de réduction de leurs émissions prévus à l'article 3 » (UNFCCC, 1998 : 3-5).

Le MDP reflète l'idée selon laquelle pour réduire le coût économique de la réduction domestique des EGES, les pays développés financent, à moindre coût, la réduction des EGES dans les pays en développement, en y transférant des technologies peu polluantes par comparaison avec celles en cours d'utilisation dans ces pays en développement (Doelle, 2008 ; Denis 2007 ; Barral 2006). Il s'agit aussi d'un nouveau mécanisme de coopération associant les pays en développement aux pays développés pour faire en sorte que l'ensemble des Etats participe à la lutte contre le changement climatique (Tsayem 2009a ; Hall et al., 2008).

Les alinéas de l'article 12 du Protocole de Kyoto précisent les principes de mise en œuvre du MDP : participation volontaire des pays ayant ratifié le Protocole de Kyoto, approbation des projets par tous les partenaires concernés, projets ne pouvant être réalisés que dans des pays qui ne figurent pas dans l'annexe 1, certification des réductions des émissions, quantification et vérification de la réduction des émissions, caractère additionnel de la réduction des émissions, émissions évitées attribuées aux pays investisseurs (pays développés), retombées socioéconomiques pour les pays hôtes (pays en développement), admission des investisseurs tant publics que privés, etc.

Le caractère additionnel de la réduction des émissions constitue la condition de «l'additionnalité » des réductions des EGES. C'est un critère fondamental du MDP. Cette condition de l'additionnalité signifie que le projet doit permettre une réduction effective des EGES, c'est-à-dire que les émissions évitées ou réduites grâce au MDP ne doivent pas se substituer, dans les pays en développement, aux émissions qui auraient pu être réduites ou évitées en l'absence du dispositif MDP. Le caractère effectif ou additionnel de la réduction des émissions est validé par comparaison avec un scénario de référence qui représente l'évolution des émissions dans l'hypothèse où tout se passe comme d'habitude (« business as usual ») (Tsayem, 2009a). En d'autres termes, il faut démontrer ou apporter la preuve que la réduction des émissions grâce au projet MDP n'aurait pas pu être obtenue en l'absence de ce projet. 
Le principe du MDP peut être résumé de manière suivante : un pays développé (ou une entreprise de ce pays développé) investit dans un projet ou finance un projet dans un pays en développement afin que la réalisation de ce projet aboutisse à une réduction des EGES. Le financement ou l'investissement doit comporter en principe un transfert de technologie devant permettre la réduction des EGES. Le pays développé (ou l'entreprise de ce pays développé) reçoit des unités de réduction certifiée des émissions (URCE), c'est-à-dire des crédits carbone, correspondant aux émissions évitées ou réduites grâce au projet MDP qu'il a financé. Ce pays développé (ou l'entreprise de ce pays développé) peut utiliser ces URCE au titre de son engagement de réduction des EGES dans le cadre du Protocole de Kyoto. Ces URCE peuvent aussi être commercialisées dans les marchés carbone.

La $7^{\text {ème }}$ conférence des parties (réunion annuelle appelée COP), rassemblant les pays ayant ratifié la Convention des Nations unies sur les changements climatiques, a précisé et adopté en 2001 (Accords de Marrakech) les modalités pratiques de fonctionnement du MDP. Les organismes de supervision et de mise en œuvre des projets, ainsi que les procédures à suivre et les critères à respecter, ont été établis et diffusés, de même que les méthodes à utiliser dans les phases d'ingénierie des projets. Les secteurs environnementaux concernés par les projets MDP sont nombreux : amélioration de l'efficacité énergétique pour les usages finaux, amélioration de l'efficacité énergétique pour l'offre d'énergie, énergies renouvelables, substitution de combustibles, agriculture (réduction des émissions de méthane et de protoxyde d'azote), procédés industriels (réduction du gaz carbonique dû au ciment, réduction des émissions fugitives liées aux halocarbures et à l'hexafluorure de soufre etc.), afforestation-reforestation (absorption ou séquestration du carbone), etc.

La démarche de formalisation et de validation des projets comporte 7 principales étapes (figure 1). Après l'élaboration du projet par ses porteurs, il est soumis à l'approbation des pays concernés. C'est l'Autorité Nationale Désignée (AND) qui délivre l'approbation dans chaque pays concerné. Chacun des pays qui participent au MDP a créé une AND au titre de l'application du Protocole de Kyoto. L'approbation préliminaire des projets par les autorités du pays hôte (pays en développement) est une condition préalable, d'autant plus que, conformément aux Accords de Marrakech, il revient à chaque pays hôte de vérifier et de valider le fait que les projets contribueront ou non au développement durable ${ }^{8}$.

\footnotetext{
${ }^{8}$ Il est étonnant de remarquer que dans le montage des projets MDP, alors que le volet environnemental (réduction des EGES) fait l'objet d'une évaluation et d'une certification validées par des organismes indépendants sous la supervision du conseil exécutif du MDP, le volet socio-économique (contribution au développement durable des pays en développement) est laissé à l'appréciation et à l'approbation de chaque pays hôte. Une autre réserve formulée à l'égard du MDP concerne la difficulté du respect et de la vérification de l'additionnalité des réductions des EGES. Par ailleurs, bien que les Accords de Marrakech stipulent que le financement public des projets MDP par les pays développés doit se faire en dehors des engagements de ces pays en matière d'aide publique au développement $(0,7 \%$ du PIB), rien ne permet de vérifier que les pays développés n'utilisent pas l'argent de l'aide publique au développement pour financer des projets MDP, ce qui correspondrait alors au détournement de l'aide publique au développement (Denis, 2007).
} 
Le Bureau Canadien du MDP et de l'Application Conjointe est l'Autorité Nationale Canadienne désignée pour approuver (ou non) les projets MDP impliquant le Canada ${ }^{9}$. Les projets approuvés sont ensuite soumis pour évaluation et validation par une Entité Opérationnelle Désignée (OED). Les EOD sont des bureaux d'études et autres cabinets d'expertises qui ont reçu l'agrément des AND et du conseil exécutif du MDP pour expertiser de manière indépendante les projets MDP qui leur sont soumis. Les projets validés par les EOD sont ensuite acceptés et enregistrés formellement par le conseil exécutif du MDP. La phase opérationnelle de ces projets peut alors commencer. Durant cette phase opérationnelle, les projets font l'objet de suivi et de vérification de leur fonctionnement censé conduire à la réduction des EGES. A l'issue de la vérification, le conseil exécutif du MDP attribue des URCE (crédits carbone) aux investisseurs (entreprises ou pays développés ayant financé le projet).

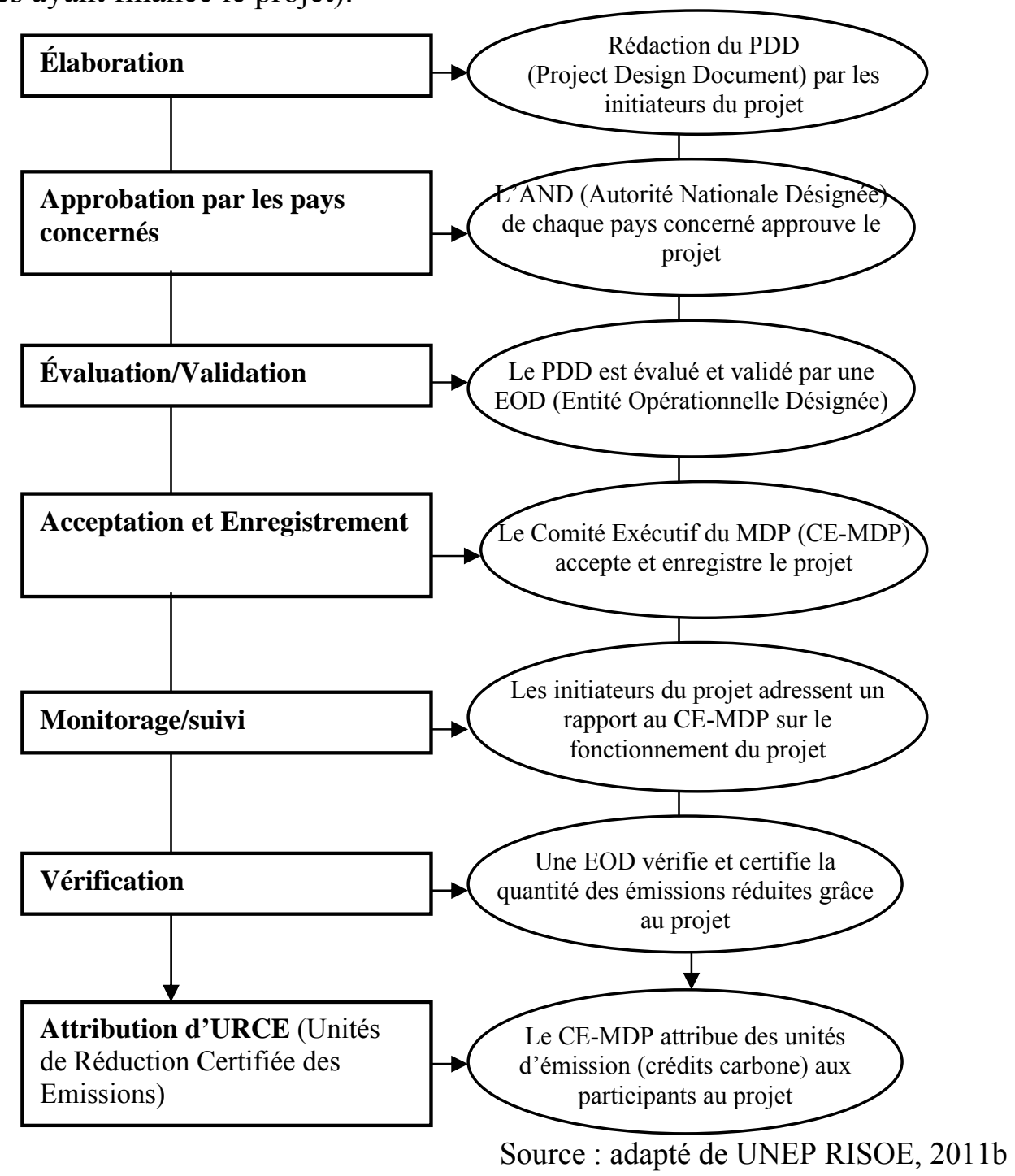

Figure 1. Démarche de mise en œuvre d'un projet MDP

\footnotetext{
${ }^{9}$ Le Bureau canadien du MDP et de l'application conjointe est basé au ministère des Affaires étrangères et du Commerce international. Il a publié sur son site Internet les règles et les critères d'approbation des projets MDP qui lui sont soumis (Ministère des Affaires étrangères et du Commerce international, 2011).
} 


\section{2. État global du MDP : vue d'ensemble des projets}

Bien que le Protocole de Kyoto (qui institue le MDP) ait été adopté en 1997, c'est avec les Accords de Marrakech en 2001 (COP 6) que l'attention accordée au MDP s'est accrûe du fait que ces Accords ont permis l'élaboration des aspects opérationnels du MDP. C'est aussi à la suite de ces Accords que les structures de fonctionnement du MDP ont été effectivement mises en place (conseil exécutif du MDP, AND, EOD, etc.). L'ingénierie des projets (procédures, cahiers des charges, etc.) s'est progressivement développée. Dès lors, le dispositif MDP a commencé à susciter de l'enthousiasme, et les premiers projets ont été acceptés en 2003 par le conseil exécutif du MDP. La création des marchés carbone, en particulier celui de l'Union européenne en 2005, a ensuite revigoré le MDP. Les projets ont alors connu un foisonnement dans les pays en développement (Tsayem, 2009a).

Les données obtenues auprès du RISOE Centre ${ }^{10}$ permettent de fournir un état général du MDP (tableau 1). Ces données indiquent qu'au premier janvier 2011, 5760 projets avaient été répertoriés dans le dispositif MDP. Parmi ces 5760 projets, 2703 avaient déjà été acceptés par le conseil exécutif du MDP et 844 projets avaient déjà donné lieu à la délivrance d'URCE ${ }^{11}$. Le secteur de l'hydro-électricité (construction de barrages par exemple) comporte 801 projets acceptés, soit 29\% des projets acceptés. Quant au secteur de l'énergie éolienne, il comporte 515 projets acceptés, soit 19\% des projets acceptés. Les projets acceptés représentent 418776 k.t.éq. $\mathrm{CO}_{2}$ par an. Les projets les plus importants en termes d'URCE sont ceux du secteur HFC (HydroFluoroCarbures). Les 21 projets HFC acceptés représentent 19\% des URCE des projets acceptés, alors que les 801 projets hydro-électriques acceptés représentent 18\% des URCE des projets acceptés (tableau 1). Les 515 projets du secteur de l'énergie éolienne représentent 12\% des URCE des projets acceptés.

Parmi les 844 projets pour lesquels 496178 k.t.éq. $\mathrm{CO}_{2}$ d'URCE ont été délivrées, $25 \%$ sont du secteur de l'hydro-électricité, $22 \%$ sont du domaine de l'énergie éolienne et $2 \%$ sont du domaine des HFC (tableau 1). Près de la moitié des URCE délivrées (49\%) l'ont été pour les 18 projets du secteur HFC, alors que les 215 projets du secteur hydro-électrique ne représentent que $5 \%$ des URCE délivrées, tout comme les 181 projets du secteur de l'énergie éolienne. Les projets dans le secteur HFC sont les plus pourvoyeurs d'URCE. Les projets dans le secteur $\mathrm{N}_{2} \mathrm{O}$ apparaissent aussi comme étant très pourvoyeurs d'URCE, puisque les 22 projets de ce domaine ont donné lieu à l'attribution de $23 \%$ de la totalité des URCE délivrées (tableau 1). Le fait que le secteur HFC soit très pourvoyeur d'URCE est sans doute dû au fort potentiel de réchauffement global des HFC, ce pouvoir de réchauffement global étant en lien avec la durée de vie des GES dans l'atmosphère. Par exemple, la durée de vie des HFC dans l'atmosphère est de 260 ans, alors qu'elle est de 12 ans pour le méthane et de 114 ans pour les $\mathrm{N}_{2} \mathrm{O}$ (GIEC, 2007).

\footnotetext{
${ }^{10}$ Des échanges ont ensuite eu lieu avec des chercheurs du RISOE Centre afin d'affiner le traitement de ces données et d'expliciter les unités utilisées pour représenter les quantités d'EGES associées aux projets.

${ }^{11} 1$ URCE équivaut à 1 tonne équivalent $\mathrm{CO}_{2}$
} 


\begin{tabular}{|c|c|c|c|c|c|c|c|c|}
\hline \multirow[b]{2}{*}{ type de projet } & \multicolumn{4}{|c|}{ projets acceptés } & \multicolumn{3}{|c|}{ URCE attribuées } & \multirow[b]{2}{*}{$\begin{array}{c}\% \\
\text { URCE }\end{array}$} \\
\hline & nombre & $\begin{array}{c}\% \\
\text { nombre }\end{array}$ & $\begin{array}{l}\text { URCE } \\
\text { (k.t.éq. } \\
\mathrm{CO}_{2} \text { ) } \\
\text { /an }\end{array}$ & $\begin{array}{c}\% \\
\text { URCE }\end{array}$ & $\begin{array}{l}\text { nombre } \\
\text { de } \\
\text { projets }\end{array}$ & $\begin{array}{c}\% \\
\text { nombre }\end{array}$ & $\begin{array}{l}\text { URCE } \\
\text { (k.t.éq. } \\
\mathrm{CO}_{2} \text { ) }\end{array}$ & \\
\hline Afforestation & 3 & 0,11 & 43 & 0,01 & 0 & 0,00 & 0 & 0,00 \\
\hline Agriculture & 0 & 0,00 & 0 & 0,00 & 0 & 0,00 & 0 & 0,00 \\
\hline $\begin{array}{l}\text { énergie issue de la } \\
\text { biomasse }\end{array}$ & 317 & 11,73 & 18677 & 4,46 & 130 & 15,40 & 16650 & 3,36 \\
\hline Ciment & 19 & 0,70 & 3214 & 0,77 & 8 & 0,95 & 1321 & 0,27 \\
\hline utilisation de $\mathrm{CO}_{2}$ & 2 & 0,07 & 24 & 0,01 & 1 & 0,12 & 10 & 0,00 \\
\hline $\begin{array}{l}\text { méthane, houille, } \\
\text { mine }\end{array}$ & 37 & 1,37 & 16562 & 3,95 & 12 & 1,42 & 3763 & 0,76 \\
\hline $\begin{array}{l}\text { distribution de } \\
\text { l'énergie }\end{array}$ & 2 & 0,07 & 67 & 0,02 & 0 & 0,00 & 0 & 0,00 \\
\hline $\begin{array}{l}\text { approvisionnement } \\
\text { des ménages en } \\
\text { électricité }\end{array}$ & 18 & 0,67 & 549 & 0,13 & 0 & 0,00 & 0 & 0,00 \\
\hline $\begin{array}{l}\text { industrie de l'énergie } \\
\text { électrique }\end{array}$ & 59 & 2,18 & 1774 & 0,42 & 26 & 3,08 & 1384 & 0,28 \\
\hline $\begin{array}{l}\text { production de } \\
\text { l'énergie électrique }\end{array}$ & 155 & 5,73 & 24426 & 5,83 & 64 & 7,58 & 21542 & 4,34 \\
\hline $\begin{array}{l}\text { service de l'énergie } \\
\text { électrique }\end{array}$ & 5 & 0,18 & 59 & 0,01 & 1 & 0,12 & 6 & 0,00 \\
\hline $\begin{array}{l}\text { offre en énergie } \\
\text { électrique }\end{array}$ & 22 & 0,81 & 4893 & 1,17 & 7 & 0,83 & 425 & 0,09 \\
\hline $\begin{array}{l}\text { substitution des } \\
\text { combustibles fossiles }\end{array}$ & 54 & 2,00 & 29402 & 7,02 & 31 & 3,67 & 7842 & 1,58 \\
\hline émissions fugitives & 17 & 0,63 & 10960 & 2,62 & 2 & 0,24 & 4600 & 0,93 \\
\hline Géothermie & 11 & 0,41 & 2779 & 0,66 & 5 & 0,59 & 684 & 0,14 \\
\hline HFC & 21 & 0,78 & 81696 & 19,51 & 18 & 2,13 & 247906 & 49,96 \\
\hline Hydro-électricité & 801 & 29,63 & 76181 & 18,19 & 215 & 25,47 & 26207 & 5,28 \\
\hline enfouissement de gaz & 179 & 6,62 & 30471 & 7,28 & 51 & 6,04 & 11619 & 2,34 \\
\hline $\begin{array}{l}\text { évitement de } \\
\text { méthane }\end{array}$ & 344 & 12,73 & 12855 & 3,07 & 66 & 7,82 & 6897 & 1,39 \\
\hline $\mathrm{N}_{2} \mathrm{O}$ & 63 & 2,33 & 47992 & 11,46 & 22 & 2,61 & 117985 & 23,78 \\
\hline PFC et SF6 & 9 & 0,33 & 3259 & 0,78 & 1 & 0,12 & 79 & 0,02 \\
\hline Reforestation & 15 & 0,55 & 606 & 0,14 & 0 & 0,00 & 0 & 0,00 \\
\hline énergie solaire & 30 & 1,11 & 570 & 0,14 & 1 & 0,12 & 1 & 0,00 \\
\hline Energie marémotrice & 1 & 0,04 & 315 & 0,08 & 0 & 0,00 & 0 & 0,00 \\
\hline Transport & 4 & 0,15 & 322 & 0,08 & 2 & 0,24 & 201 & 0,04 \\
\hline énergie éolienne & 515 & 19,05 & 51081 & 12,20 & 181 & 21,45 & 27055 & 5,45 \\
\hline Total & 2703 & 100,00 & 418776 & 100,00 & 844 & 100,00 & 496178 & 100,00 \\
\hline
\end{tabular}

kilotonne d'équivalent $\mathrm{CO}_{2}$, soit 1000 tonnes d'équivalent $\mathrm{CO}_{2}$

$\mathrm{CO}_{2}$ : gaz carbonique ; HFC : HydroFluoroCarbures ; $\mathrm{N}_{2} \mathrm{O}$ : protoxyde d'azote (oxydes nitreux) ; PFC : perfluorocarbures ; SF6 : Hexafluorure de soufre

Source : adapté de UNEP RISOE, 2011a

Tableau 1. Vue d'ensemble des projets MDP (au 01/01/2011)

Les données du conseil exécutif du MDP (UNFCCC, 2011) permettent de caractériser davantage la répartition des projets MDP (tableau 2). La nomenclature que ce conseil exécutif utilise pour répartir les projets montre que plus de la moitié des projets $(64 \%)$ relèvent du domaine des 
industries énergétiques ${ }^{12}$ basées essentiellement sur les énergies renouvelables, alors que 15\% des projets relèvent du domaine du traitement et de l'élimination des déchets (photo 1). Mais comme le montre le tableau 1, le grand nombre de projets dans les domaines des énergies renouvelables ne signifie pas une grande quantité d'URCE. Il existe une très faible quantité de projets dans les secteurs des transports, de la production des métaux et de la foresterie. D’après Barral (2006), la rentabilité financière des projets est le principal élément qui oriente le choix du secteur d'activités dans lequel des projets sont mis en place. Cela signifie que les industries énergétiques et le traitement des déchets sont des domaines dans lesquels il est possible d'obtenir une grande quantité de crédits carbone, sans doute du fait que les technologies ou les procédés industriels des investisseurs des pays développés sont déjà bien éprouvés dans ces domaines.

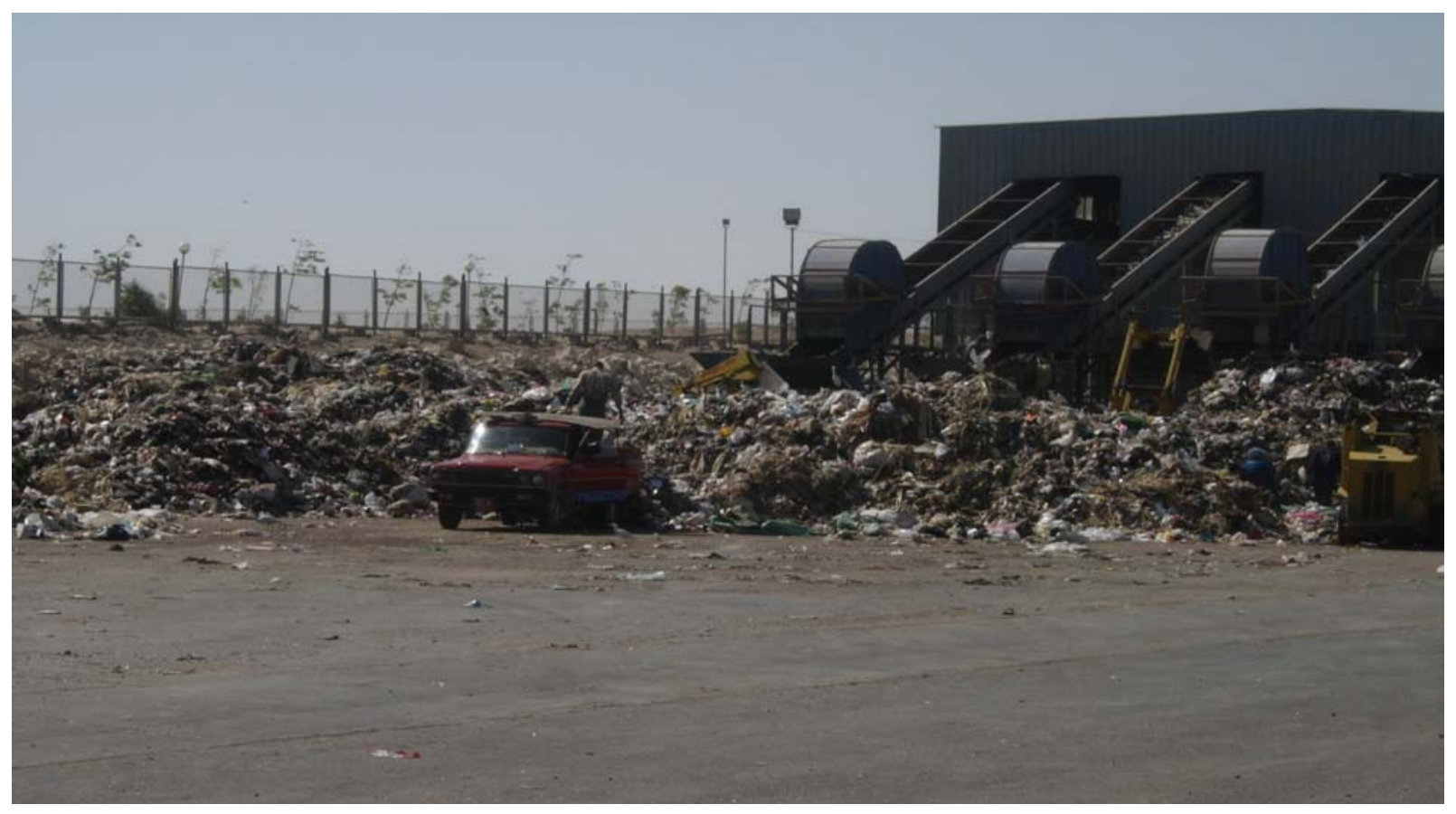

Photo 1. Dépôt d'ordures dont le traitement par l'entreprise ENTAG-ECARU fait partie d'un projet MDP au Caire (Égypte). Cliché Tsayem, mai 2009

\footnotetext{
12 le nucléaire ne donne pas lieu à la délivrance d'URCE dans le cadre du MDP
} 


\begin{tabular}{lcc}
\hline domaines & nombre de projets & \% nombre de projets \\
\hline $\begin{array}{l}\text { Industries énergétiques (essentiellement les } \\
\text { énergies renouvelables) }\end{array}$ & 2118 & $\mathbf{6 4 , 9 1}$ \\
\hline Traitement et élimination des déchets & 506 & 15,51 \\
\hline Industries manufacturières & 154 & 4,72 \\
\hline $\begin{array}{l}\text { Émissions fugitives de carburants (solide, } \\
\text { pétrole et gaz) }\end{array}$ & 153 & 4,69 \\
\hline Agriculture & 134 & 4,11 \\
\hline Industries chimiques & 69 & 2,11 \\
\hline Extraction/production minérale & 38 & 1,16 \\
\hline Demande énergétique & 36 & 0,74 \\
\hline $\begin{array}{l}\text { Émissions fugitives liées aux halocarbures et } \\
\text { à l'hexafluorure de soufre }\end{array}$ & 24 & 0,58 \\
\hline Afforestation et reforestation & 19 & 0,25 \\
\hline Production de métaux & 8 & 0,12 \\
\hline Transport & 4 & $\mathbf{1 0 0 , 0 0}$ \\
\hline total & 3263 & 10 \\
\hline N.B. un projet peut appartenir à plusiurs & & \\
\hline
\end{tabular}

N.B. un projet peut appartenir à plusieurs domaines, ce qui explique que le total soit de 3263 projets

Source : UNFCCC, 2011a

\section{Tableau 2. Répartition des projets acceptés suivant la nomenclature du conseil exécutif du MDP (au 14/01/2011)}

Les données du conseil exécutif MDP font état de 2754 projets acceptés au 14 janvier 2011 (tableau 3). Avec respectivement $42 \%$ et $22 \%$ de l'ensemble des projets acceptés, la Chine et l'Inde figurent en tête des pays hôtes (figure 2). La totalité des projets acceptés représente globalement un volume annuel de 511605902 tonnes équivalent $\mathrm{CO}_{2}$. D'après les estimations du conseil exécutif MDP, les projets acceptés pourront donner lieu, jusqu'en 2012, à la délivrance de près de 2 milliards de tonnes équivalent $\mathrm{CO}_{2}$ (UNFCCC, 2011a). Les projets concernant la Chine représentent plus de la moitié (53\%) des URCE, alors que ceux qui concernent l'Inde et le Brésil représentent respectivement $16 \%$ et $9 \%$ des URCE (tableau 3 et figure 2). Ces 3 pays concentrent au total $70 \%$ de l'ensemble des projets acceptés, ce qui représente près de $80 \%$ des crédits carbone engendrés par les projets MDP acceptés. Les pays d'Asie et du Pacifique (80\% des projets acceptés), suivis de ceux d'Amérique latine et des Caraïbes (18\% des projets acceptés), sont particulièrement actifs dans la mise en œuvre des projets MDP, contrairement aux pays d'Afrique (moins de $2 \%$ des projets acceptés). Plusieurs facteurs expliquent la très faible quantité de projets MDP en Afrique (Desanker, 2005) : complexité et coûts élevés du montage des projets, manque de capacités techniques et institutionnelles nationales, etc. La très faible quantité de projets MDP en Afrique a amené la Banque Mondiale à faire une étude prospective du marché du MDP dans ce continent. Les résultats de cette étude, portant sur un panel de 44 pays de l'Afrique subsaharienne, révèlent un potentiel de plus de 3200 projets MDP dans plusieurs secteurs d'activités (De Gouvello et al., 2008). 


\begin{tabular}{|c|c|c|c|c|}
\hline Pays & $\begin{array}{l}\text { nombre de } \\
\text { projets }\end{array}$ & $\begin{array}{l}\text { \% nombre } \\
\text { de projets }\end{array}$ & $\begin{array}{l}\text { URCE } \\
\text { (t.éq.COO }{ }_{2} \text { )/an }\end{array}$ & $\begin{array}{l}\% \\
\text { URCE }\end{array}$ \\
\hline Chine & 1174 & 42,63 & 275197038 & 53,79 \\
\hline Inde & 606 & 22,00 & 84869828 & 16,59 \\
\hline Brésil & 184 & 6,68 & 47693975 & 9,32 \\
\hline Mexique & 125 & 4,54 & 7323755 & 1,43 \\
\hline Malaisie & 87 & 3,16 & 743439 & 0,15 \\
\hline Indonésie & 55 & 2,00 & 688548 & 0,13 \\
\hline République de Corée & 51 & 1,85 & 62718384 & 12,26 \\
\hline Philippines & 48 & 1,74 & 159023 & 0,03 \\
\hline Viet Nam & 46 & 1,67 & 4499098 & 0,88 \\
\hline Chili & 42 & 1,53 & 5140813 & 1,00 \\
\hline Thaïlande & 41 & 1,49 & 815224 & 0,16 \\
\hline Colombie & 26 & 0,94 & 883223 & 0,17 \\
\hline Pérou & 23 & 0,84 & 472867 & 0,09 \\
\hline Argentine & 20 & 0,73 & 4405251 & 0,86 \\
\hline Israël & 18 & 0,65 & 632238 & 0,12 \\
\hline Afrique du Sud & 18 & 0,65 & 1794261 & 0,35 \\
\hline Honduras & 16 & 0,58 & 469903 & 0,09 \\
\hline Equateur & 15 & 0,54 & 863835 & 0,17 \\
\hline Guatemala & 11 & 0,40 & 918588 & 0,18 \\
\hline Pakistan & 10 & 0,36 & 2314762 & 0,45 \\
\hline Autres pays & 138 & 5,01 & 9001849 & 1,76 \\
\hline Total & 2754 & 100,00 & 511605902 & 100,00 \\
\hline
\end{tabular}

Source : UNFCCC 2011a

Tableau 3. Répartition des projets acceptés auxquels participent les pays non annexe 1 (au 14/01/2011)

Les pays émergents, en croissance économique rapide, canalisent une grande majorité de projets MDP en liaison avec des investissements en provenance des pays développés. Ces pays émergents profitent ainsi de leur «boom économique » et de leur fort potentiel démographique (vastes marchés de consommation) pour attirer les investissements MDP et les transferts de technologie venant des pays développés (Denis, 2007). 


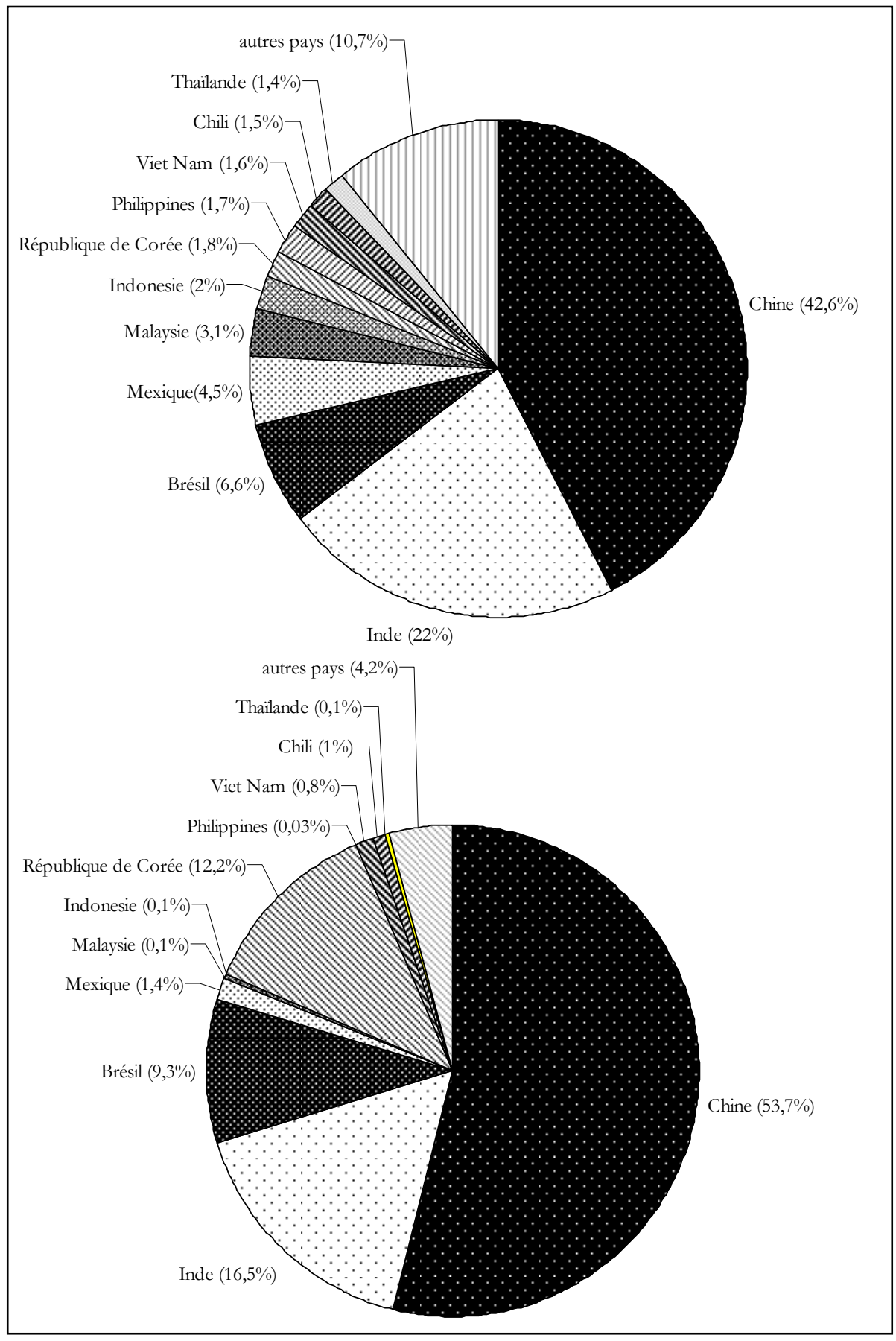

Figure 2. Répartition des projets MDP acceptés (en haut) et des crédits carbone (en bas) associés à ces projets dans les pays en développement (au 14/01/2011)

Parmi les pays de l'annexe 1, le Royaume Uni de Grande Bretagne et d'Irlande du Nord (914 projets acceptés, soit 29\% des projets acceptés), la Suisse (601 projets acceptés, soit 19\% des projets acceptés), le Japon (368 projets acceptés, soit 11\% des projets acceptés) et les Pays-Bas (356 projets acceptés, soit $11 \%$ des projets acceptés) se distinguent par le nombre de projets 
auxquels ils participent ${ }^{13}$ (tableau 4 et figure 3 ). Ces 4 pays sont parmi les pays ayant les plus forts engagements de réduction des EGES dans le cadre du protocole de Kyoto (12,5\% pour la Grande Bretagne, 8\% pour la Suisse, 6\% pour le Japon ainsi que pour les Pays-Bas). La participation du Canada porte sur 53 projets, soit 1,68\% des projets acceptés. Elle est quasi médiane mais faible (tableau 4 et figure 3). En collaborant avec des pays en développement par des investissements financiers dans des projets MDP, les pays développés peuvent engranger des URCE soit pour les commercialiser dans les marchés carbone, soit pour les faire comptabiliser dans leurs engagements chiffrés de réduction des EGES au titre de l'application du Protocole de Kyoto. Les projets MDP permettent en outre aux pays développés (ou aux entreprises de ces pays développés) de diffuser et de commercialiser, dans les pays en développement, les technologies dites propres ou économes en énergie.

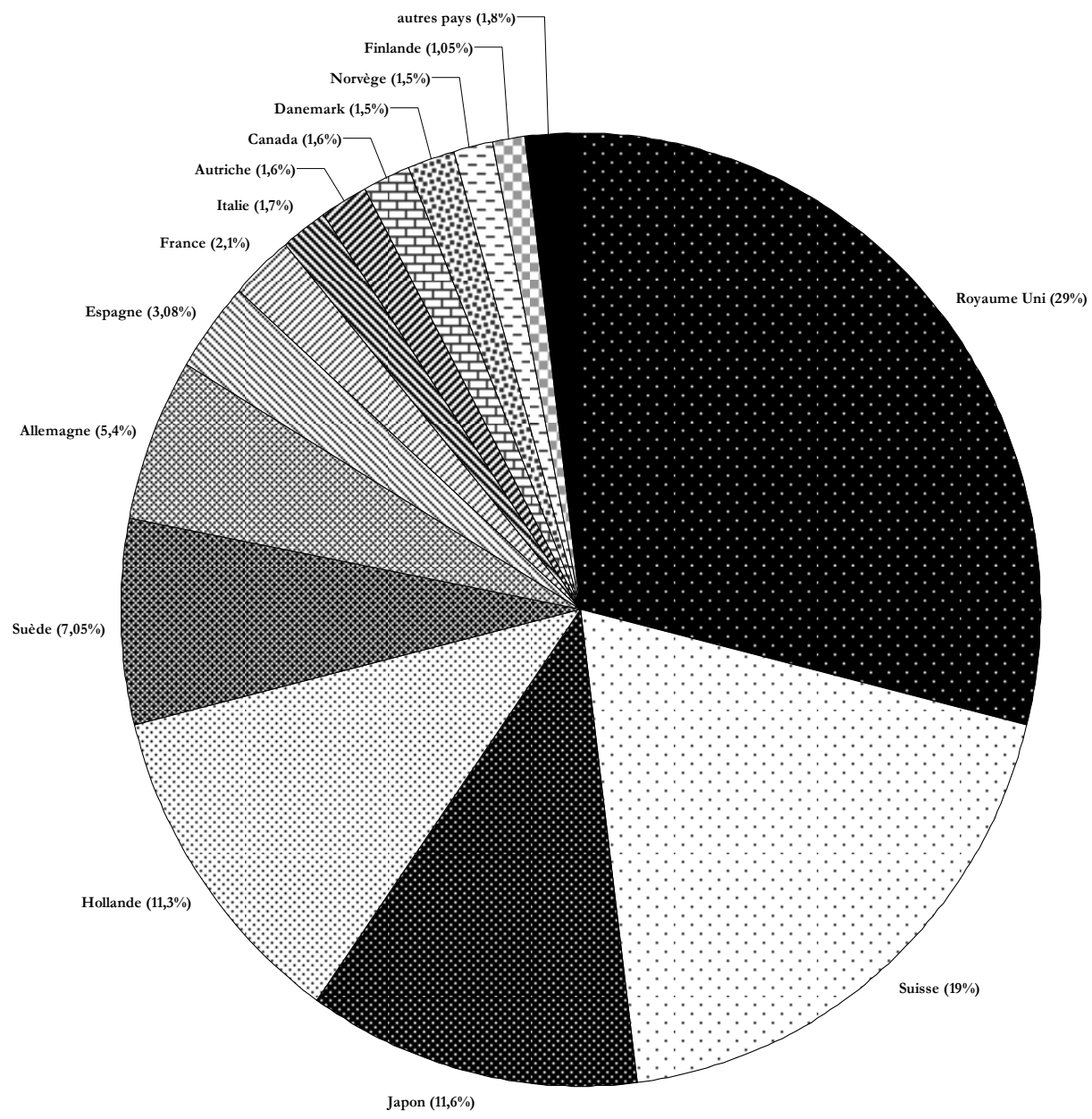

Figure 3. Participation des pays développés au MDP (en \% des projets acceptés au 14/01/2011)

\footnotetext{
${ }^{13}$ Plusieurs pays de l'annexe 1peuvent participer à un même projet dans un pays hôte, ce qui explique que le nombre total de projets auxquels participent ces pays de l'annexe 1 (3148) soit supérieur au nombre de projets dans les pays hôtes (2754) au 14 janvier 2011.
} 


\begin{tabular}{|c|c|c|}
\hline Pays & $\begin{array}{l}\text { nombre de } \\
\text { projets }\end{array}$ & $\begin{array}{l}\text { \% nombre } \\
\text { de projets }\end{array}$ \\
\hline Royaume Uni & 914 & 29,03 \\
\hline Suisse & 601 & 19,09 \\
\hline Japon & 368 & 11,69 \\
\hline Pays-Bas & 356 & 11,31 \\
\hline Suède & 222 & 7,05 \\
\hline Allemagne & 173 & 5,49 \\
\hline Espagne & 97 & 3,08 \\
\hline France & 67 & 2,13 \\
\hline Italie & 54 & 1,71 \\
\hline Autriche & 53 & 1,68 \\
\hline Canada & 53 & 1,68 \\
\hline Danemark & 50 & 1,59 \\
\hline Norvège & 49 & 1,56 \\
\hline Finlande & 33 & 1,05 \\
\hline Belgique & 25 & 0,79 \\
\hline Luxembourg & 20 & 0,64 \\
\hline Portugal & 9 & 0,29 \\
\hline Irlande & 2 & 0,06 \\
\hline Australie & 1 & 0,03 \\
\hline Liechtenstein & 1 & 0,03 \\
\hline Total & 3148 & 100,00 \\
\hline
\end{tabular}

N.B. Plusieurs pays de l'annexe 1 peuvent participer à un même projet dans un pays non annexe 1 . Dans ce cas, le projet est multiplié par le nombre de pays de l'annexe 1 qui participent au projet. C'est ce qui explique que le nombre total des projets soit 3148 et non 2 754. Dans le cas où plusieurs pays de l'annexe 1 participent à un même projet, les URCE sont partagées entre ces pays de l'annexe 1.

Source : UNFCCC, 2011a

Tableau 4. Répartition des projets acceptés auxquels participent les pays de l’annexe 1 (au 14/01/2011)

Une étude récente sur le transfert de technologies dans les secteurs environnementaux concernés par les projets MDP répertoriés par le conseil exécutif du MDP au 30 juin 2010 montre que ce transfert de technologie est envisagé dans $30 \%$ à $44 \%$ des projets, représentant environ $50 \%$ des URCE prévues (UNFCCC, 2010a). Cette étude indique une baisse continue de la quantité de projets comportant un transfert de technologies dans les pays en développement. D'après cette étude, les technologies transférées dans $5 \%$ des projets sont d'origine canadienne, alors qu'elles sont d'origine allemande pour $17 \%$ des projets et d'origine japonaise pour $10 \%$ des projets.

\section{Géographie de la participation du Canada au MDP}

Après avoir ratifié le Protocole de Kyoto en décembre 2002, le Canada a mis en place des structures pour remplir ses engagements au titre de ce Protocole (création de l'Autorité Nationale Désignée qui est le Bureau Canadien du MDP et de l'Application Conjointe, création du registre 
national canadien du Protocole de Kyoto qui est basé à Environnement Canada, etc.). Dès lors, l'Etat canadien a commencé à participer au MDP, en délivrant des autorisations et des approbations aux entreprises publiques et privés (de droit canadien) intéressées par des projets MDP dans les pays en développement.

Pour faire une esquisse géographique de la participation du Canada au MDP, une requête a été effectuée sur le site Internet du conseil exécutif du MDP le 14 janvier 2011 (UNFCCC, 2011a), afin d'extraire les données relatives aux projets MDP impliquant le Canada en tant que pays annexe 1. Seuls les projets déjà acceptés par le conseil exécutif du MDP ont été extraits. Les résultats montrent que le Canada participe à 53 projets acceptés (tableau 5). Répartis entre 23 pays, ces 53 projets représentent 38774725 tonnes équivalent $\mathrm{CO}_{2}$ prévues par an, soit 7,5\% des URCE annuelles que représentent les projets acceptés. Ces 53 projets ont été enregistrés entre août 2005 et janvier 2011. Bien que sa participation soit faible en termes de nombre de projets MDP acceptés (tableaux 4 et 5), le Canada apparaît comme un pays intermédiaire ou médian, par rapport aux autres pays de l'annexe 1 (comme le Royaume Uni ou la Suisse). Avec 8 projets (15\% du total), la Chine est le pays ayant le plus grand nombre de projets acceptés auxquels le Canada participe. Après la Chine, il y a la Malaisie (7 projets, 13\% du total) et le Brésil (6 projets, $11 \%$ du total). Les 8 projets acceptés concernant la Chine représentent $86 \%$ des URCE de l'ensemble des projets acceptés auxquels le Canada participe, alors que les 7 projets concernant la Malaisie ne représentent que 2\% de l'ensemble des URCE (tableau 5).

La répartition géographique des projets acceptés auxquels le Canada participe (figure 4) montre une prépondérance de la présence du Canada dans des projets MDP en Amérique latine (Brésil, Argentine, Chili, etc.) et en Asie (Chine, Malaisie, Népal, etc.), bien que le Canada soit présent ailleurs, notamment en Afrique (Ouganda, Afrique du Sud, Egypte, etc.). Reflétant considérablement sa situation dans les relations internationales (Boniface et Védrine, 2010 ; Guellec, 1999 ; Kirton, 1996), la participation du Canada dans le dispositif MDP traduit aussi l'importance et le poids croissant des pays émergents, et en particulier la Chine, dans les relations internationales actuelles, notamment en ce qui concerne l'environnement et la lutte contre les changements climatiques (Dahan et al., 2011 ; Audet et Bonin, 2010).

Adepte du multilatéralisme et des institutions onusiennes, membre de la francophonie et du Commonwealth, le Canada, à travers ses entreprises impliquées dans les projets MDP approuvés par le gouvernement Canadien représenté par l'AND, participe au dispositif international du MDP en se déployant dans le monde, en tirant parti de sa proximité avec l'Amérique latine et en s'inscrivant dans la dynamique économique et environnementale des pays émergents comme la Chine (figure 5). Ce type de déploiement géographique relatif au MDP se fait aussi dans d'autres cadres de coopération géopolitique et environnementale qui ne portent pas spécifiquement sur le MDP. Par exemple, depuis 2007, le Canada est membre du Partenariat Asie-Pacifique sur le développement propre et le climat. C'est une initiative internationale qui regroupe sept pays (Canada, Australie, Chine, Inde, Japon, Corée du Sud et Etats-Unis), avec pour objectif de faire collaborer les secteurs publics et privés en vue d'accroître la mise au point, le déploiement et la diffusion des technologies énergétiques propres (sécurité énergétique, réduction de la pollution atmosphérique et atténuation des changements climatiques) tout en favorisant une croissance économique durable et la réduction de la pauvreté (Gouvernement du Canada, 2011). Dans le cadre de ce partenariat, le Canada retrouve les États-unis, qui ne participent pas au MDP du fait qu'ils n'ont pas ratifié le Protocole de Kyoto. 


\begin{tabular}{|c|c|c|c|c|}
\hline pays hôte & $\begin{array}{l}\text { nombre de } \\
\text { projets }\end{array}$ & $\begin{array}{l}\text { \% nombre } \\
\text { de projets }\end{array}$ & $\begin{array}{l}\text { URCE prévues } \\
\left.\text { (t.éq.CO } \mathrm{O}_{2}\right) / \text { an }\end{array}$ & $\begin{array}{l}\text { \% URCE } \\
\text { prévues }\end{array}$ \\
\hline Honduras & 1 & 1,9 & 37032 & 0,1 \\
\hline Chili & 4 & 7,5 & 490378 & 1,3 \\
\hline Pérou & 1 & $\overline{1,9}$ & 13845 & 0,0 \\
\hline Brésil & 6 & 11,3 & 614149 & 1,6 \\
\hline Népal & 2 & 3,8 & 93883 & 0,2 \\
\hline Argentine & 3 & 5,7 & 798785 & 2,1 \\
\hline Moldavie & 4 & 7,5 & 226585 & 0,6 \\
\hline Costa Rica & 1 & 1,9 & 6431 & 0,0 \\
\hline Salvador & 1 & 1,9 & 183725 & 0,5 \\
\hline Colombie & 1 & 1,9 & 18028 & 0,0 \\
\hline Chine & 8 & 15,1 & 33425990 & 86,2 \\
\hline Malaisie & 7 & 13,2 & 1075964 & 2,8 \\
\hline Philippines & 2 & 3,8 & 62846 & 0,2 \\
\hline Indonésie & 2 & 3,8 & 614163 & 1,6 \\
\hline Guatemala & 1 & 1,9 & 118527 & 0,3 \\
\hline Afrique du Sud & 1 & 1,9 & 68833 & 0,2 \\
\hline Ouganda & 2 & 3,8 & 41774 & 0,1 \\
\hline Ethiopie & 1 & 1,9 & 29343 & 0,1 \\
\hline Albanie & 1 & 1,9 & 22964 & 0,1 \\
\hline Egypte & 1 & 1,9 & 430350 & 1,1 \\
\hline Inde & 1 & 1,9 & 14162 & 0,0 \\
\hline Cuba & 1 & 1,9 & 342235 & 0,9 \\
\hline Guyana & 1 & 1,9 & 44733 & 0,1 \\
\hline Total & 53 & 100 & 38774725 & 100 \\
\hline
\end{tabular}

Tableau 5. Répartition géographique des projets MDP acceptés auxquels le Canada participe (au 14/01/2011)

Une autre requête a été effectuée le 14 janvier 2011 sur le site Internet du conseil exécutif du MDP pour extraire les projets MDP auxquels participe le Canada et pour lesquels des URCE ont déjà été délivrées (UNFCCC, 2011b). Les résultats de cette requête indiquent que le Canada participe à 14 projets pour lesquels des URCE ont été délivrées (tableau 6). Ces 14 projets représentent $1,6 \%$ de l'ensemble des projets pour lesquels des URCE ont été attribuées par le conseil exécutif du MDP. Parmi ces 14 projets qui concernent 9 pays en développement, 3 sont situés au Brésil, 3 en Chine, 2 en Malaisie, etc. Pour ces 14 projets, 49426000 tonnes équivalent $\mathrm{CO}_{2}$ ont été délivrées, soit $9,9 \%$ des URCE déjà délivrées. 93\% des URCE délivrées l'ont été pour les 3 projets en Chine, alors que pour le projet en Argentine et pour les 3 projets au Brésil, $2,7 \%$ et $1,7 \%$ des URCE ont été délivrées respectivement (tableau 6 ). 


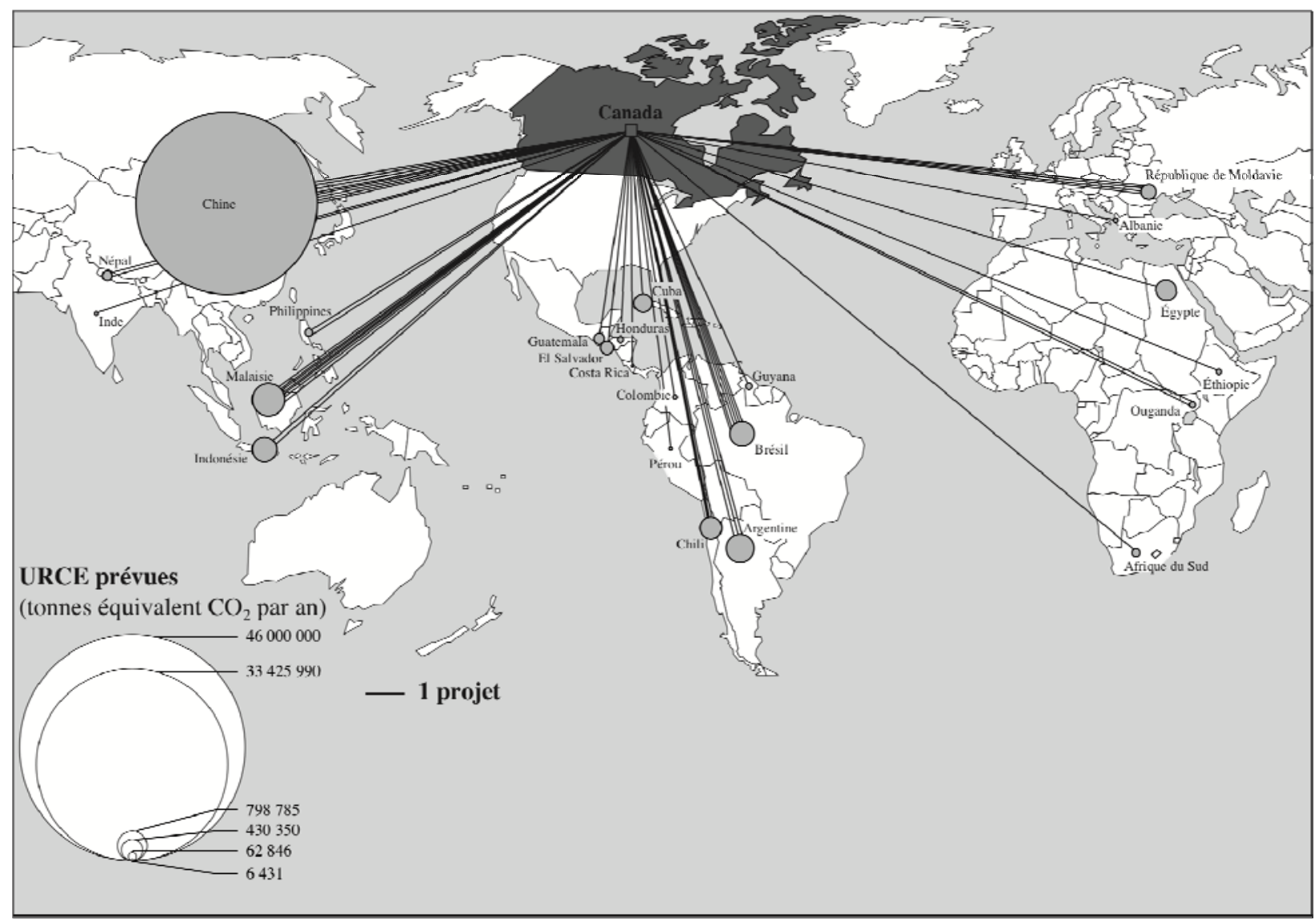

Figure 4. Géographie de la participation du Canada aux projets MDP acceptés (au 14/01/2011)

\begin{tabular}{|c|c|c|c|c|}
\hline pays hôte & $\begin{array}{l}\text { nombre de } \\
\text { projets }\end{array}$ & $\begin{array}{l}\text { \% nombre } \\
\text { de projets }\end{array}$ & $\begin{array}{l}\text { URCE obtenues } \\
\text { (t.éq. } \mathrm{CO}_{2} \text { ) }\end{array}$ & $\begin{array}{l}\text { \% URCE } \\
\text { obtenues }\end{array}$ \\
\hline Salvador & 1 & 7,1 & 216000 & 0,4 \\
\hline Brésil & 3 & 21,4 & 859000 & 1,7 \\
\hline Colombie & 1 & 7,1 & 82000 & $\overline{0,2}$ \\
\hline Guatemala & 1 & 7,1 & 397000 & 0,8 \\
\hline Chine & 3 & 21,4 & 46047000 & 93,2 \\
\hline Indonésie & 1 & 7,1 & 81000 & 0,2 \\
\hline Malaisie & 2 & 14,3 & 227000 & 0,5 \\
\hline Cuba & 1 & 7,1 & 167000 & 0,3 \\
\hline Argentine & 1 & 7,1 & 1350000 & 2,7 \\
\hline Total & 14 & 100,0 & 49426000 & 100,0 \\
\hline
\end{tabular}

Tableau 6. Répartition géographique des projets MDP auxquels le Canada participe et pour lesquels des URCE ont été délivrées (au 14/01/2011)

La répartition géographique des projets auxquels le Canada participe et qui ont donné lieu à la délivrance des crédits carbone (figure 5) confirme la présence du Canada surtout dans les projets MDP en Amérique latine (Brésil, Argentine, etc.) et en Asie (Chine, Malaisie, etc.). Cette 
répartition géographique montre qu'en Afrique il n'y a aucun projet MDP auquel le Canada participe et pour lequel des crédits carbone ont été délivrés (figure 5). Cette observation illustre la marginalité de l'Afrique dans le dispositif MDP (Desanker, 2005 ; Tsayem, 2009a).

D'après l'Autorité Nationale Désignée (AND) ${ }^{14}$ du Canada, le gouvernement du Canada ne participe pas au MDP en tant qu'acteur direct ou porteur de projets. Il instruit les dossiers et donne l'approbation, le cas échéant, pour que les projets associant les entreprises de droit canadien, que ce soit des entreprises publiques ou privées, soient ensuite acceptés par le conseil exécutif du MDP et donne lieu à la délivrance de crédits carbone (URCE) que les entreprises de droit canadien peuvent ensuite utiliser ou commercialiser dans les marchés carbone ${ }^{15}$. Cette observation suggère que la distribution géographique des projets MDP auxquels le Canada participe n'est pas la résultante d'une volonté géopolitique ou diplomatique, ni le résultat d'une stratégie de politique étrangère ou internationale du gouvernement Canadien. Le fait que ce soit les entreprises, et non les États, qui élaborent et mettent en œuvre des projets MDP pour engranger des crédits carbone, à condition que les États approuvent ces projets MDP, indique que la signification géopolitique et diplomatique de la distribution des projets MDP peut être secondaire, surtout pour les pays développés, dans la mesure où les entreprises de ces pays développés, qui participent aux projets MDP dans des pays en développement, peuvent considérer le MDP comme un outil d'atténuation de leurs contraintes en matière d'EGES. Vu sous cet angle, le MDP apparaît comme un outil de délocalisation des réductions des EGES, le coût de la réduction des EGES étant moins élevé dans les pays en développement que dans les pays développés (Denis, 2007 ; Barral, 2006).

\footnotetext{
${ }^{14}$ qui est le Bureau Canadien du MDP et de l'Application Conjointe

${ }^{15}$ communication personnelle lors d'un entretien le 16 juillet 2010 au Bureau Canadien du MDP et de l'Application Conjointe
} 


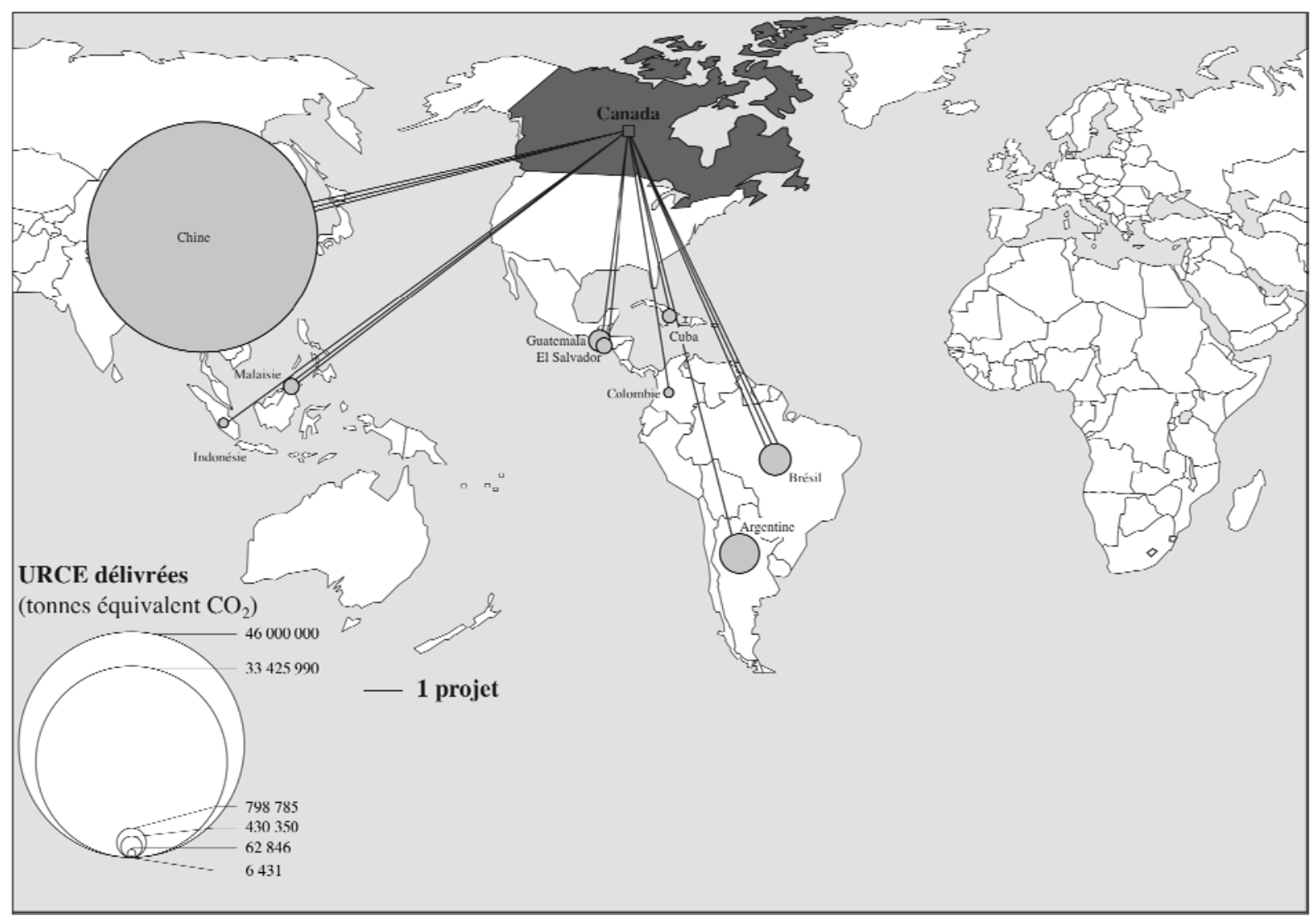

Figure 5. Géographie de la participation du Canada aux projets MDP pour lesquels des crédits carbone ont été délivrés (au 14/01/2011)

\section{Profil environnemental de la participation du Canada au MDP}

Pour esquisser le profil environnemental de la participation du Canada au MDP, les secteurs environnementaux concernés par les 53 projets acceptés auxquels le Canada participe ont été décomptés et répertoriés (tableau 7). La répartition de ces projets suivant les secteurs environnementaux, conformément à la nomenclature du conseil exécutif du MDP, montre une prédominance des projets dans le secteur des industries énergétiques (essentiellement les énergies renouvelables, par exemple les éoliennes). À l'exception des projets au Salvador, en Indonésie, en Ethiopie, en Albanie et en Inde, les projets dans les autres pays concernent le secteur des industries énergétiques. C'est donc dans ce domaine environnemental que les entreprises canadiennes exportent ou transfèrent la technologie ${ }^{16}$ dans le plus grand nombre de pays qui accueillent des projets MDP acceptés auxquels elles participent. Le secteur du traitement et de l'élimination des déchets constitue le deuxième secteur environnemental en termes de nombre de projets, suivi par les secteurs de la foresterie (afforestation et/ou reforestation) et de l'agriculture. Le secteur de la demande énergétique ne concerne qu'un projet (en Moldavie), ainsi que le secteur des émissions fugitives liées aux halocarbures et à l'hexafluorure de soufre (un projet en Chine), et le secteur des industries chimiques (un projet en Chine).

\footnotetext{
${ }^{16}$ la technologie ainsi transférée ou exportée n'est pas nécessairement canadienne ; elle peut provenir ou appartenir à un autre pays de l'annexe 1, mais pas d'un pays en développement, conformément aux Accords de Marrakech.
} 


\begin{tabular}{|c|c|c|c|c|c|c|c|c|c|c|}
\hline pays hôte & nombre de projets & URCE prévues & 1 & 3 & 4 & 5 & 11 & 13 & 14 & 15 \\
\hline Honduras & 1 & 37032 & $\sqrt{ }$ & & & & & & & \\
\hline Chili & 4 & 490378 & $\sqrt{ }$ & & & & & $\sqrt{ }$ & & $\sqrt{ }$ \\
\hline Pérou & 1 & 13845 & $\sqrt{ }$ & & & & & & & \\
\hline Brésil & 6 & 614149 & $\sqrt{ }$ & & $\sqrt{ }$ & & & $\sqrt{ }$ & $\sqrt{ }$ & $\sqrt{ }$ \\
\hline Népal & 2 & 93883 & $\sqrt{ }$ & & & & & & & \\
\hline Argentine & 3 & 798785 & $\sqrt{ }$ & & & & & $\sqrt{ }$ & & \\
\hline Moldavie & 4 & 226585 & $\sqrt{ }$ & $\sqrt{ }$ & & & & & $\sqrt{ }$ & \\
\hline Costa Rica & 1 & 6431 & $\sqrt{ }$ & & & & & & & \\
\hline Salvador & 1 & 183725 & & & & & & $\sqrt{ }$ & & \\
\hline Colombie & 1 & 18028 & $\sqrt{ }$ & & & & & & & \\
\hline Chine & 8 & 33425990 & $\sqrt{ }$ & & & $\sqrt{ }$ & $\sqrt{ }$ & $\sqrt{ }$ & & $\sqrt{ }$ \\
\hline Malaisie & 7 & 1075964 & $\sqrt{ }$ & & & & & $\sqrt{ }$ & & $\sqrt{ }$ \\
\hline Philippines & 2 & 62846 & $\sqrt{ }$ & & & & & $\sqrt{ }$ & & \\
\hline Indonésie & 2 & 614163 & & & $\sqrt{ }$ & & & & & \\
\hline Guatemala & 1 & 118527 & $\sqrt{ }$ & & & & & & & \\
\hline Afrique du Sud & 1 & 68833 & $\sqrt{ }$ & & & & & $\sqrt{ }$ & & \\
\hline Ouganda & 2 & 41774 & $\sqrt{ }$ & & & & & & $\sqrt{ }$ & \\
\hline Ethiopie & 1 & 29343 & & & & & & & $\sqrt{ }$ & \\
\hline Albanie & 1 & 22964 & & & & & & & $\sqrt{ }$ & \\
\hline Egypte & 1 & 430350 & $\sqrt{ }$ & & $\sqrt{ }$ & & & & & \\
\hline Inde & 1 & 14162 & & & $\sqrt{ }$ & & & & & \\
\hline Cuba & 1 & 342235 & $\sqrt{ }$ & & & & & & & \\
\hline Guyana & 1 & 44733 & $\sqrt{ }$ & & & & & & & \\
\hline
\end{tabular}

Les chiffres en gras représentent les secteurs environnementaux

1 : industries énergétiques (sources renouvelables / non renouvelables)

3: demande énergétique

4 : industries manufacturières

5 : industries chimiques

11 : émissions fugitives liées aux halocarbures et à l'hexafluorure de soufre

13 : traitement et élimination des déchets

14 : afforestation et reforestation

15 : agriculture

Source : UNFCCC, 2011a

Tableau 7. Secteurs environnementaux concernés par les projets MDP acceptés auxquels participe le Canada (au 14/01/2011)

Le même exercice a été effectué pour les 14 projets auxquels le Canada participe et qui ont donné lieu à la délivrance des crédits carbone (tableau 8). Les résultats confirment la prédominance du secteur des industries énergétiques, suivi par le secteur du traitement et de l'élimination des déchets. Deux projets dans le secteur de l'agriculture ont donné lieu à la délivrance de crédits carbone, alors qu'un seul projet, respectivement dans le domaine des industries énergétiques et dans celui des industries manufacturières, a donné lieu à la délivrance des crédits carbone, tout comme dans le domaine des émissions fugitives liées aux halocarbures et à l'hexafluorure de soufre (tableau 8). Le tableau 9 résume les informations relatives à un projet MDP auquel le Canada participe dans le secteur du traitement et de l'élimination des déchets. 


\begin{tabular}{|l|r|r|l|l|l|l|l|l|}
\hline pays hôte & nombre de projets & $\begin{array}{l}\text { URCE } \\
\text { obtenues }\end{array}$ & $\mathbf{1}$ & $\mathbf{4}$ & $\mathbf{5}$ & $\mathbf{1 1}$ & $\mathbf{1 3}$ & $\mathbf{1 5}$ \\
\hline Salvador & 1 & 216000 & & & & & $\sqrt{ }$ & \\
\hline Brésil & 3 & 859000 & $\sqrt{ }$ & & & & $\sqrt{ }$ & $\sqrt{ }$ \\
\hline Colombie & 1 & 82000 & $\sqrt{ }$ & & & & & \\
\hline Guatemala & 1 & 397000 & $\sqrt{ }$ & & & & & \\
\hline Chine & 3 & 46047000 & $\sqrt{ }$ & & $\sqrt{ }$ & $\sqrt{ }$ & & \\
\hline Indonésie & 1 & 81000 & & $\sqrt{ }$ & & & & \\
\hline Malaisie & 2 & 227000 & $\sqrt{ }$ & & & & $\sqrt{ }$ & $\sqrt{ }$ \\
\hline Cuba & 1 & 167000 & $\sqrt{ }$ & & & & & \\
\hline Argentine & 1 & 1350000 & & & & & $\sqrt{ }$ & \\
\hline
\end{tabular}

Les chiffres en gras représentent les secteurs environnementaux

1 : industries énergétiques (sources renouvelables / non renouvelables)

4 : industries manufacturières

5 : industries chimiques

11 : émissions fugitives liées aux halocarbures et à l'hexafluorure de soufre

13 : traitement et élimination des déchets

15 : agriculture

Source : UNFCCC, 2011b

\section{Tableau 8. Secteurs environnementaux concernés par les projets MDP auxquels le Canada} participe et qui ont donné lieu à la délivrance de crédits carbone (au 14/01/2011)

\footnotetext{
Projet d'enfouissement des déchets à González Catán et Ensenada (province de Buenos Aires, municipalités de Matanza et d'Ensenada, Argentine)

Référence du projet : 426

Projet accepté le 17 juin 2006

URCE délivrées : 1350000 tonnes équivalent $\mathrm{CO}_{2}$

Participants au projet :

-Argentine (pays hôte) Miguel A. Barrera - Estudio Contable

-Canada (Conestoga-Rovers \& Associates Limited, promoteur du projet, entreprise privée)

- Royaume-Uni (BGC International, organisme privé)

- Royaume-Uni (IXIS Environnement \& Infrastructures, entreprise privée)

- Suisse (participation indirecte, European Carbon Fund)

Le projet est développé par Conestoga-Rovers \& Associates Limited pour deux décharges situées à González Catán et Ensenada. Ces décharges reçoivent des déchets solides non dangereux, municipaux, industriels, commerciaux, institutionnels et certains déchets agricoles depuis plus de 20 ans. La décomposition de ces déchets fait que les deux décharges émettent du dioxyde de carbone $\left(\mathrm{CO}_{2}\right)$ et du méthane $\left(\mathrm{CH}_{4}\right)$. Le projet comprend la construction de systèmes de gaz d'enfouissement composé d'une grille de tranchées horizontales et verticales d'extraction de gaz, de ventilateurs centrifuges, et des supports mécaniques et soussystèmes électriques, ainsi que des accessoires nécessaires pour recueillir les gaz d'enfouissement. Le but du projet est de collecter et de traiter des déchets dans les décharges de González Catán et de Ensenada, pendant 10 ans, en utilisant une technologie de haute efficacité, pour réduire les EGES. Le projet prévoit de réduire des EGES équivalent à 7698095 URCE dont 5117995 à González Catán et 2580100 à Ensenada). La contribution du projet au développement durable en Argentine est multiple : environnement propre et sain pour les populations vivant aux alentours des deux décharges, réduction des risques d'incendies des décharges, réduction de la pollution des eaux souterraines, emploi de la main d'œuvre locale lors de la mise en œuvre du projet.
}

\section{Source : UNFCCC, 2004}

Tableau 9. Résumé d'un projet MDP auquel le Canada participe et qui a donné lieu à la délivrance de crédits carbone

\section{Une faible importance du MDP pour la politique canadienne de réduction des EGES}

D'après les inventaires que le Canada a communiqués au secrétariat de la Convention des Nations unies sur les changements climatiques, ses EGES étaient de $591872 \mathrm{Gg}$ équivalent $\mathrm{CO}_{2}$ en 1990, $717156 \mathrm{Gg}$ équivalent $\mathrm{CO}_{2}$ en 2000, $730990 \mathrm{Gg}$ équivalent $\mathrm{CO}_{2}$ en 2005 et 734566 $\mathrm{Gg}$ équivalent $\mathrm{CO}_{2}$ en 2008 (UNFCCC, 2010). Au moment de l'entrée en vigueur du Protocole de Kyoto en 2005, les EGES du Canada étaient déjà supérieures de $23 \%$ par rapport à celles de 
1990, ramenant alors à près de 30\% la réduction à effectuer à l'horizon 2012 par rapport à 1990. L'engagement de réduction de 6\% des EGES du Canada au titre du Protocole de Kyoto correspond à une réduction de 240 millions de tonnes équivalent $\mathrm{CO}_{2}$ par an entre 2008 et 2012 par rapport aux EGES de l'année 1990. Dans l'hypothèse ${ }^{17}$ où les crédits carbones délivrés pour les projets MDP acceptés auxquels le Canada participe sont effectivement et entièrement attribués au Canada, ces crédits représenteraient environ $20 \%$ de la quantité annuelle des EGES que le Canada est censée réduire au titre de l'application du Protocole de Kyoto.

Arrivé au pouvoir en 2006 et reconduit en 2011, 1'actuel gouvernement du Canada ${ }^{18}$ a émis des critiques et des réserves sur le Protocole de Kyoto, considérant qu'il était déséquilibré (du fait qu'il n'exige pas aux pays émergents de réduire leurs EGES), irréaliste et pénalisant pour le développement du Canada ${ }^{19}$. Il n'est dès lors pas étonnant que la Loi de mise en œuvre du Protocole de Kyoto, adoptée par le Canada en 2007 et mise à jour en 2010 (GOUVERNEMENT DU CANADA, 2010a ), ne mentionne pas explicitement le MDP comme élément de la politique canadienne de réduction de ses EGES. Il en est de même pour le Plan d'action du Canada en matière de changements climatiques (GOUVERNEMENT DU CANADA, 2010b), ou du Plan sur les changements climatiques aux fins de la Loi de mise en œuvre du Protocole de Kyoto (GOUVERNEMENT DU CANADA, 2010c). Ces documents de planification sont portés essentiellement sur les mesures domestiques au niveau national et au niveau provincial (réduction des consommations d'énergie, réglementation sur les émissions, réduction de l'intensité carbone, réduction de la pollution, financements pour l'innovation et pour le développement de technologies "vertes », amélioration de l'efficacité énergétique des véhicules, des transports et des bâtiments, taxes carbone, etc.). Le gouvernement du Canada n'affiche pas l'intérêt qu'il pourrait avoir en utilisant le MDP comme contribution à la réduction de ses EGES ${ }^{20}$.

\section{Conclusion}

En instituant le MDP, le Protocole de Kyoto a offert la possibilité que les crédits carbone générés par ce mécanisme soient utilisés par les Etats de l'annexe 1 (et par les entreprises de ces Etats) dans le cadre de leurs engagements de réduction de leurs EGES. En quelques années de fonctionnement, le MDP est devenu un important pourvoyeur de crédits carbone pour les marchés et les transactions au niveau international (Gardette et Locatelli, 2007 ; Murphy et al., 2009). Le MDP est non seulement un instrument international de lutte contre le changement climatique, mais aussi un instrument de promotion d'un développement moins émetteur de GES dans les pays en développement (Denis, 2007 ; Barral, 2006 ; Tsayem, 2009a).

D’après les estimations prévisionnelles du conseil exécutif du MDP, les émissions évitées d'ici

\footnotetext{
${ }^{17} \mathrm{Il}$ s'agit ici d'une hypothèse de réflexion, dans la mesure où le gouvernement du Canada, jusqu'à présent, n'utilise pas les crédits carbones issus du MDP, et ne les comptabilise donc pas dans ses engagements de réduction des EGES au titre du Protocole de Kyoto ; le gouvernement du Canada préfère que ces crédits soient utilisés par des entreprises de droit canadien (d'après l'entretien et les échanges que nous avons eus en juillet 2010 avec le Bureau Canadien du MDP et de l'Application Conjointe). Par ailleurs, les projets MDP auxquels le Canada participe et qui ont donné lieu à la délivrance de crédits carbone associent généralement d'autres pays de l'annexe 1, de sorte que ces crédits ne reviennent pas en totalité au Canada (ou aux entreprises canadiennes) mais à l'ensemble des pays annexe 1 participant à ces projets.

${ }_{10}^{18}$ Gouvernement dirigé par le premier ministre Stephen Harper.

${ }^{19}$ d'après l'entretien que nous avons eu le 15 juillet 2010 avec un expert travaillant pour la Fondation David Suzuki

${ }^{20}$ cette observation découle de l'entretien que nous avons eu le 15 juillet avec un expert travaillant pour la Fondation David Suzuki.
} 
2012 grâce aux projets MDP acceptés au 14 janvier 2011 (2 751 projets) seront d'un volume de près de 2 milliards de tonnes équivalent $\mathrm{CO}_{2}$, soit environ $36 \%$ de l'effort de réduction des EGES des pays de l'annexe 1. Avec 2,7 milliards de tonnes équivalent $\mathrm{CO}_{2}$ attendus en 2012, la totalité des projets répertoriés par le conseil exécutif du MDP au 4 janvier 2011 représenterait alors 51\% de l'effort de réduction des EGES des pays de l'annexe 1. Ainsi, grâce au MDP, la moitié des 5\% de réduction prescrite par le Protocole de Kyoto est possible non pas par des réductions à l'intérieur des pays de l'annexe 1, mais par des réductions dans les pays en développement. Pour éviter que des réductions des EGES soient réalisées de manière très importante dans des pays en développement, au détriment des réductions dans les pays développés, l'Union européenne a proposé que le recours au MDP, comme aux autres mécanismes de flexibilité, soit une alternative utilisée de manière subsidiaire (principe dit de supplémentarité) par rapport aux efforts domestiques de chacun des pays développés tenus de limiter ses EGES (Yamin, 2000, cité par Denis, 2007).

Bien que le Canada ne soit pas un acteur majeur du MDP, si on considère le nombre de projets auxquels il est associé (1,6\% des projets acceptés et 9,9\% des URCE délivrées), le recours à ce mécanisme aurait pu être un complément ou un appoint par rapport à ses efforts de réduction domestique des EGES. Or le gouvernement du Canada semble ne pas considérer le MDP comme un instrument pour remplir ses engagements en matière de réduction des EGES en vertu du Protocole de Kyoto. Ce gouvernement donne l'impression d'utiliser le MDP moins comme un outil de sa participation internationale aux efforts de réduction des EGES. Il semble d'utiliser davantage comme un outil de facilitation de la participation des entreprises de droit canadien aux projets pouvant leur permettre d'obtenir des crédits carbone à l'étranger.

Le Canada a participé activement à l'élaboration et à l'adoption, en 2009, de l'Accord de Copenhague dans lequel il a fait inscrire l'engagement de réduire ses EGES de 17\% à l'horizon 2020 par rapport à 2005 (Dahan et al., 2011 ; Audet et Bonin, 2010 ; De Perthuis et Delbosc, 2010). Cette baisse de 17\% signifie que le Canada s'engage à ce qu'en 2020, ses EGES soient de $606721 \mathrm{Gg}$ équivalent $\mathrm{CO}_{2}$ (puisqu'elles étaient de $730990 \mathrm{Gg}$ équivalent $\mathrm{CO}_{2}$ en 2005), soit une hausse de 2,5\% par rapport à ses EGES de 1990 (alors que son engagement dans le Protocole de Kyoto porte sur une réduction de 6\% entre 2008 et 2012 par rapport à 1990). En s'engageant dans l'Accord de Copenhague, le Canada s'est aligné sur la position des Etats-Unis, alors que la participation du Canada au MDP lui confère une particularité géo-environnementale et géopolitique par rapport aux Etats-Unis. Consolidé en 2010 avec l'Accord de Cancun, l'Accord de Copenhague a été considéré par le gouvernement du Canada comme le cadre dans lequel il souhaite désormais inscrire sa politique et ses actions internationales de lutte contre le changement climatique, tout en n'excluant pas l'éventualité de faire appel à un MDP reformé et élargi ${ }^{21}$. Il a annoncé en décembre 2011, lors de la conférence des parties à Durban, qu'il ne participerait pas à une deuxième phase d'application du Protocole de Kyoto censée commencer en 2013. En matière de stratégie internationale de réduction des EGES, le gouvernement canadien inscrit sa politique dans le cadre du multilatéralisme et d'une plus grande coopération avec les États-unis, privilégiant ainsi une coopération internationale en dehors du cadre prévu par le Protocole de Kyoto.

\footnotetext{
${ }^{21}$ cette observation découle de l'entretien que nous avons eu le 16 juillet à Environnement Canada avec des personnes qui représentent le Canada dans les négociations internationales sur la lutte contre les changements climatiques.
} 


\section{Références bibliographiques}

AUDET R., BONIN P. 2011. Les accords de Cancun face aux enjeux des négociations internationales sur le climat. Vertigo, la revue électronique en sciences de l'environnement (en ligne), débats et perspectives, 2011, mis en ligne le 7 février 2011, consulté le 20 mai 2011.

Banque Mondiale, 2009, Développement et changement climatique. Pour un climat favorable au développement. Rapport sur le développement dans le monde 2010, abrégé-version préliminaire. Consulté sur Internet (http://siteresources.worldbank.org/INTWDR2010/Resources/52876781226014527953/Overview-French.pdf) le 7 février 2011.

BARRAL Jean Pierre, 2006, « Aider les pays du Sud à lutter contre leurs émissions de gaz à effet de serre : le mécanisme de développement propre prévu par le protocole de Kyoto est-il la solution?» dans S. MICHAILOF (dir.), In A quoi sert d'aider le Sud? Paris, Economica : 271-297.

BONIFACE P., VÉDRINE H., 2010. Atlas du monde global. Paris, A. Colin, 125 p.

DAHAN A., BUFFET C., VIARD-CRETAT A. 2011. L'ère post-Copenhague du régime climatique. Le compromis de Cancun: vertu du pragmatisme ou masque de l'immobilisme? Rapport de recherche, Centre A. Koyré.

De Gouvello Christophe, Dayo Félix, Thioye Massamba, 2008, Projets énergétiques propres pour le développement de l'Afrique subsaharienne. Relever le potentiel, éliminer les barrières. Rapport de la Banque Mondiale, résumé à l'attention des décideurs. Consulté sur

Internet (http://siteresources.worldbank.org/INTSENEGALINFRENCH/Resources/4615841175072268436/ResumeRapportProjetenergetiquesproprespourdeveloppementAfriquesub saharienne.pdf) le 7 février 2011.

DE PERTHUIS C., DELBOSC A. 2010. Négociations climatiques: les enjeux du postCopenhague. L'Economie Politique, n ${ }^{\circ}$ 46, p. 70-81.

DENIS Benjamin, 2007, « Le mécanisme pour un développement propre. Un instrument politique au service du développement et de la préservation du climat? », dans P. CORNUT, T. BAULER, E. ZACCAI (dir.), Environnement et inégalités sociales, Bruxelles, Éditions de l'Université de Bruxelles : 157-170.

DESANKER Paul, 2005, « Le protocole de Kyoto et le MDP en Afrique : une bonne idée mais... », Unasylva, $\mathrm{n}^{\circ} 222$, vol.56:24-26.

DOELlE Meinhard, 2008, "Global carbon trading and climate change mitigation in Canada: options for the use of the Kyoto mechanisms", in S. BERnSteIn, J. BRUnNEe, D. DUFF, A. GREEN (dir.), A globally integrated climate policy for Canada, Toronto, University of Toronto Press: 79-202.

Gardette Yves Marie, Locatelli Bruno, 2007, Les marchés du carbone forestier. Comment un projet forestier peut-il vendre du carbone ? Rapport ONI et CIRARD. Consulté sur Internet (http://www.cbfp.org/tl_files/archive/thematique/marches_carbone_forestier.pdf) le 7 février 2011.

GIEC, 2007, Changements climatiques 2007. Rapport de synthèse. Consulté sur Internet (http://www.ipcc.ch/pdf/assessment-report/ar4/syr/ar4_syr_fr.pdf) le 7 février 2011. 
Gouvernement du Canada 2011, Partenariat Asie-Pacifique sur le développement propre et le climat. Consulté sur Internet (http:/www.climatechange.gc.ca/papapp/default.asp?lang=Fr\&n=FFB91B5D-1) le 8 février 2011.

Gouvernement Du CANADA, 2010a, Loi de mise en oeuvre du Protocole de Kyoto. Consulté sur Internet (http://lois-laws.justice.gc.ca) le 2 février 2011.

Gouvernement Du CANADA, 2010b, Plan d'action du Canada en matière de changements climatiques. Consulté sur Internet (http://climatechange.gc.ca/default.asp?lang=Fr\&n=D43918F1-0) le 2 février 2011.

GOUVERNEMENT DU CANADA, 2010c, Plan sur les changements climatiques aux fins de la loi de mise en ouvre du Protocole de Kyoto. Consulté sur Internet (http://climatechange.gc.ca/default.asp?lang=Fr\&n=4D57AF05-1) le 2 février 2011.

GUELLEC J., 1999. Atlas de 1'espace mondial. Paris, Ellipses, 192 p.

Hall Daniel, Levi Michael, Pizer William, Ueno Takahiro, 2008, Policies for developing country engagement, Discussion paper 08-15, Harvard Kennedy School. Consulté sur Internet (http://belfercenter.ksg.harvard.edu/files/PizerWeb.pdf) le 6 février 2011.

KIRTON J., 1996. « Une ouverture sur le monde : la nouvelle politique étrangère canadienne du gouvernement Chrétien », Etudes Internationales, vol. 27, n² 2, 1996, p. 257-279.

LAVALLÉE Sophie, 2010, «Le principe des responsabilités communes mais différenciées à Rio, Kyoto et Copenhague. Essai sur la responsabilité de protéger le climat», Revue Études Internationales, vol. XLI, $\mathrm{n}^{\circ} 1: 51-78$.

MENS Yann, 2008, «Réchauffement climatique: que vont faire les pays émergents?», Alternatives Internationales, $\mathrm{n}^{\circ} 41: 48-59$.

Ministère des Affaires étrangères et du Commerce international, 2011, Exigences relatives à l'agrément d'une participation volontaire pour les projets MDP, Bureau canadien du MDP et de l'application conjointe. Consulté sur Internet (http://www.international.gc.ca/cdm-mdp/cdmar-eamdp/index.aspx?lang=fra) le 2 février 2011.

MurPHY Deborah, Drexhage John, Wooders Peter, 2009, Les mécanismes internationaux du marché du carbone au sein d'un accord post-2012 sur les changements climatiques, rapport IISD. Consulté sur Internet (http://www.iisd.org/pdf/2009/international_carbon_market_mechanisms_fr.pdf) le 7 février 2011.

PNUD 2007, La lutte contre le changement climatique : un impératif de solidarité humaine dans un monde divisé. Rapport mondial sur le développement humain 2007-2008. Consulté sur Internet (http://hdr.undp.org/en/reports/global/hdr2007-2008/chapters/french/) le 7 février 2011 .

TSAYem Demaze Moise, 2009a, « Paradoxes conceptuels du développement durable et nouvelles initiatives de coopération Nord-Sud: le Mécanisme pour un Développement Propre (MDP) », Cybergéo, Revue Européenne de Géographie, article 443. Consulté sur Internet (http://cybergeo.revues.org/index22065.html) le 7 février 2011.

TSAYem Demaze Moïse, 2009b, «Le protocole de Kyoto, le clivage Nord-Sud et le défi du développement durable », L’Espace Géographique, n² : 139-156. 
UNEP RISOE, 2011, CDM/JI Pipeline Overview. Consulté sur Internet (cdmpipeline.org/overview.htm) le 14 janvier 2011.

UNEP RISOE, 2011a, CDM/JI Pipeline Overview Page. Consulté sur Internet (http://cdmpipeline.org/overview.htm) le 18 janvier 2011.

UNEP RISOE, 2011b, Introduction au MDP: Mécanisme pour le Développement Durable. Consulté sur Internet (http://cd4cdm.org/Publications/brochure\%20french/Cdm\%20brochure\%20french.pdf), le 8 février 2011.

UNFCC 2011a, CDM Statistics. Consulté sur Internet (http://cdm.unfccc.int/Statistics/index.html) le 14 janvier 2011.

UNFCC, 2004, Project 0426 : González Catán and Ensenada Landfill Gas Project, Projet Design Document Form. Consulté sur Internet (http://cdm.unfccc.int/Projects/DB/SGSUKL1146836073.39/view) le 8 février 2011.

UNFCCC 2010b, Données présentées dans les inventaires de gaz à effet de serre pour la période 1990-2008. Consulté sur Internet (http://unfccc.int/resource/docs/2010/sbi/fre/18f.pdf) le 7 février 2011.

UNFCCC 2011b, Issuance Certified Emission Reductions (CERs). Consulté sur Internet (http://cdm.unfccc.int/Issuance/cers iss.html) le 14 janvier 2011.

UNFCCC, 1998, Protocole de Kyoto à la Convention-cadre des Nations unies sur les changements climatiques. Consulté sur le Internet (http://unfccc.int/resource/docs/convkp/kpfrench.pdf) le 2 février 2011

UNFCCC, 2010a, The contribution of the Clean Development Mechanism under the Kyoto Protocol to technology transfer. Consulté sur Internet (http://cdm.unfccc.int/Reference/Reports/TTreport/TTrep10.pdf) le 7 février 2011.

\section{Remerciements}

Cette étude a été effectuée avec l'appui du gouvernement du Canada, suite à l'obtention d'une Bourse de Recherche en Etudes Canadiennes (BREC). L'auteur remercie le gouvernement du Canada (Affaires étrangères et Commerce international Canada), ainsi que toutes les personnes qui ont accepté de le recevoir au Canada pour un entretien (Dale Marshall de la Fondation David Suzuki, Heather Low du Bureau Canadien du MDP et de l'Application Conjointe, Dany Drouin et Laurence Blandford de Environnement Canada). Il remercie aussi Stan Frederik du RISOE Centre, qui lui a transmis des données sur les projets MDP et a répondu à ses questions. 